Please do not remove this page

RMIT

UNIVERSITY

\title{
Structures for single-bit digital comb filtering
}

Sadik, Amin; Hussain, Zahir; O'Shea, Peter

https://researchrepository.rmit.edu.au/esploro/outputs/9921859506601341/filesAndLinks?institution=61RMIT_INST\&index=null

Sadik, A., Hussain, Z., \& O'Shea, P. (2005). Structures for single-bit digital comb filtering. Proceedings of the 2005 Asia-Pacific Conference on Communications.

https://researchrepository.rmit.edu.au/discovery/fulldisplay/alma9921859506601341/61RMIT_INST:Resea rchRepository

Repository homepage: https://researchrepository.rmit.edu.au

(c) 2005 IEEE. Personal use of this material is permitted. However, permission to reprint/republish this material for advertising or promotional purposes or for creating new collective works for resale or redistribution to servers or lists, or to reuse any copyrighted component of this work in other works must be obtained from the IEEE.

Downloaded On 2023/04/26 19:50:58 +1000 


\title{
Structures for Single-Bit Digital Comb Filtering
}

\author{
Amin Z. Sadik*, Zahir M. Hussain**, Senior Member, IEEE, and Peter O'Shea*** \\ * School of Engineering Systems, Queensland University of Technology, Brisbane, Australia \\ (Currently a Visiting Researcher at SECE, RMIT, Melbourne, Australia) \\ ** School of Electrical \& Computer Engineering, RMIT, Melbourne, Australia \\ *** School of Engineering Systems, Queensland University of Technology, Brisbane, Australia \\ E-mails: amin.sadik@rmit.edu.au,zmhussain@ieee.org, pj.oshea@qut.edu.au
}

\begin{abstract}
Two structures for single-bit digital comb filtering are proposed. The first structure is based on ternary filtering, however, the output of the filter is in single-bit format. The second structure is based on second-order sigma-delta modulation $(\Sigma \Delta \mathbf{M})$. Both filtering structures contain no multi-bit multiplication, making the comb filter efficient for implementation. This filter can be utilized in a wide range of promising applications.
\end{abstract}

\section{INTRODUCTION}

Comb filters found a wide range of applications such as the suppression of clutter from fixed objects in moving-target indicator radars and the rejection of power-line harmonics in the promising technology of using the power line communication (PLC) as a third pipe to deliver broadband access to home and business. Comb filters are usually constructed using multi-bit architectures. Single-bit $\Sigma \Delta$ modulation have recently received increased attention because of their good performances and efficient VLSI implementation.

In this paper, two structures for single-bit output comb filtering are proposed and simulated. The first structure is a combination of a ternary filtering stage and a $\Sigma \Delta \mathrm{M}$. The second structure is based on a second-order $\Sigma \Delta \mathrm{M}$.

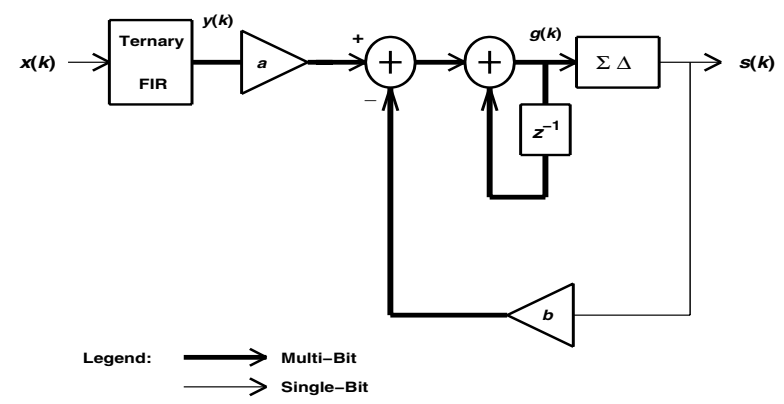

Fig. 1. Block diagram of the ternary $\Sigma \Delta$ filter.

\section{A TERnARY-Sigma-Delta COMB Filter}

The single-bit comb filter is designed according to the configuration shown in Fig.(1), which can accept both multibit and single-bit input formats. The ternary filter is an FIR filter with ternary taps (i.e., $+1,0,-1$ ) [1]. This ternary format allows a simple implementation of the FIR filter; it is most efficient when the input signal is in single-bit format. The structure of the ternary filter is shown in Fig.(2). The

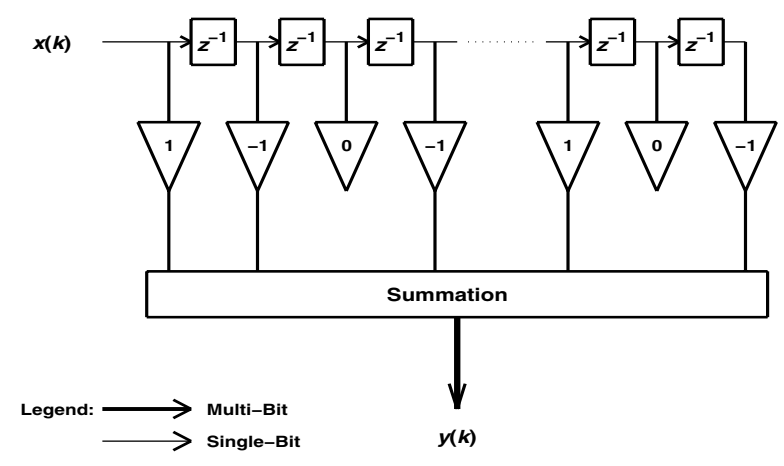

Fig. 2. Block diagram of a ternary FIR filter.

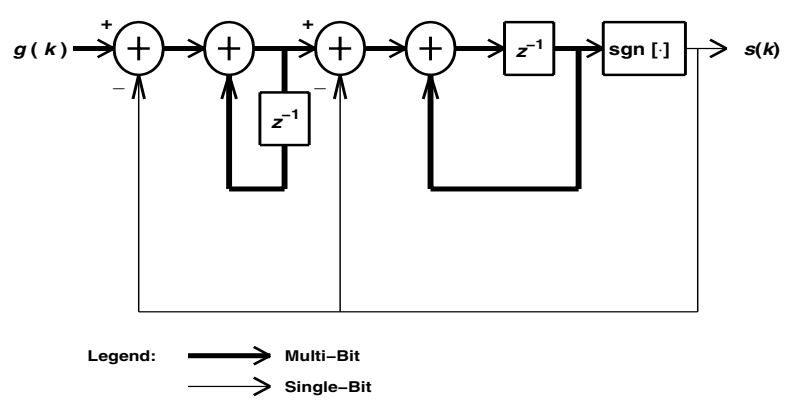

Fig. 3. Block diagram of a second-order $\Sigma \Delta \mathrm{M}$.

ternary filter output $y(k)$ is given by the convolution of ternary taps $\{h(i)\}$ (or simply $\left\{h_{i}\right\}$ ) and the input signal $\{x(k)\}$ as follows:

$$
y(k)=\sum_{i=0}^{M} h_{i} x_{k-i}
$$

where $M$ is the order of the filter. The tap values are generated via $\Sigma \Delta$ modulation of a target impulse response. The digital $\Sigma \Delta \mathrm{M}$ used for this purpose must have tri-level output, and must have a flat signal frequency response over the bandwidth of interest [2]. The ternary filter requires operation at an oversampled rate (OSR), a requirement that will be met since the input signal is assumed here to be a $\Sigma \Delta$ modulated bit-stream. The structure of the typical second-order $\Sigma \Delta \mathrm{M}$ which can be used to encode the ternary taps is shown in Fig.(3). To analyze the performance of $\Sigma \Delta \mathrm{M}$, an approximate 
quantization noise model, referred to as the input-independent additive white noise approximation, is normally used. In this linear model, the quantization noise $e(n)$ is assumed to be uniformly distributed between $-\Delta$ and $\Delta$ (where $\Delta$ is the quantization step-size) in the band of interest $\left(-f_{s} / 2 \leq f \leq\right.$ $f_{s} / 2$ ). The larger the OSR is, the better this assumption will be. Theoretically, in second-order modulator, each doubling of sampling rate achieves a signal-to-quantization-noise ratio (SQNR) improvement of about $15 \mathrm{~dB}$ [3].

The $\mathrm{z}$-domain transfer function of this $\Sigma \Delta \mathrm{M}$ model is given by:

$$
H(z)=G(z) z^{-1}+E(z)\left(1-z^{-1}\right)^{2}
$$

where $G(z)$ and $E(z)$ represent the signal and the quantization noise transfer functions, respectively. The noise shaping filter, $\left(1-z^{-1}\right)^{2}$, attenuates the quantization noise in the signal band and amplifies it outside the signal band (higher frequencies). These high-frequency noise components can be eliminated by a subsequent digital filtering that also decimates the sample rate.

From (2), the frequency response is given by:

$$
H\left(e^{j \Omega}\right)=G\left(e^{j \Omega}\right) e^{-j \Omega}+E\left(e^{j \Omega}\right)\left(1-e^{-j \Omega}\right)^{2}
$$

where $\Omega=2 \pi f / f_{s}$ is the normalized radian frequency.

The response of the overall system $H_{\mathrm{ov}}$ will be the combination of the frequency response of the ternary filter $H_{T}\left(e^{j \Omega}\right)$ and the frequency response of the IIR- $\Sigma \Delta \mathrm{M}$ filter $H_{\mathrm{IIR}}\left(e^{j \Omega}\right)$ as follows [4]:

$$
H_{\mathrm{ov}}\left(e^{j \Omega}\right)=H_{T}\left(e^{j \Omega}\right) \cdot H_{\mathrm{IIR}}\left(e^{j \Omega}\right) .
$$

From (3) and (4) we get:

$$
H_{\mathrm{ov}}\left(e^{j \Omega}\right)=H_{T}\left(e^{j \Omega}\right) \cdot\left[H_{\mathrm{IIRS}}\left(e^{j \Omega}\right)+H_{\mathrm{IIRN}}\left(e^{j \Omega}\right)\right]
$$

where $H_{\text {IIRS }}\left(e^{j \Omega}\right)$ and $H_{\text {IIRN }}\left(e^{j \Omega}\right)$ are the signal and noise parts of $H_{\mathrm{IIR}}\left(e^{j \Omega}\right)$, respectively. Now $H_{\mathrm{ov}}\left(e^{j \Omega}\right)$ can be expressed as follows:

$$
H_{\mathrm{ov}}\left(e^{j \Omega}\right)=\frac{G\left(e^{j \Omega}\right) K\left(e^{j \Omega}\right)}{D\left(e^{j \Omega}\right)}+\frac{E\left(e^{j \Omega}\right) P\left(e^{j \Omega}\right)}{D\left(e^{j \Omega}\right)}
$$

where

$$
\begin{aligned}
K\left(e^{j \Omega}\right)= & e^{-j \Omega}+e^{-2 j \Omega}(b-3)+3 e^{-3 j \Omega}-e^{-4 j \Omega} \\
D\left(e^{j \Omega}\right)= & 1-(1-a) e^{-j \Omega} \\
P\left(e^{j \Omega}\right)= & e^{-j \Omega}(b-5)+e^{-2 j \Omega}(10-2 b)+ \\
& e^{-3 j \Omega}(b-10)+5 e^{-4 j \Omega}-e^{-5 j \Omega} .
\end{aligned}
$$

noting that $a$ and $b$ are the multiplication constants shown in Fig.(1).

Experimental results in analog-to-digital conversion (ADC) indicate that the signal-to-quantization-noise ratio (SQNR) can be improved by using a ternary quantizer in the feed-back loop. The extent of improvement depends on the quantizer characteristics, the thresholds, and the output level [5].
The IIR-based filter, however, suffers from the disadvantages that the phase is no longer linear, and that the filter is more vulnerable to coefficient quantization errors than standard FIR filter.

\section{Design And Simulation of Ternary Sigma-Delta COMB FilTER}

The steps to design a ternary filter can be summarized as follows [6]:

1) Generate the FIR filter coefficients that satisfies the required specifications using Remez Exchange algorithm.

2) Interpolate the FIR filter coefficients by a factor of OSR (to oversample the target impulse response to the desired OSR). Several techniques can be used, such as spline, FFT, linear, and cubic. Spline method is adopted here.

3) Ternary encode the filter coefficients, where a set of ternary-valued coefficients are generated from the interpolated filter coefficients.

4) Upsample the input signal by a factor of OSR.

5) Remodulate the output of the ternary filter (eq.1) to single-bit format using $\Sigma \Delta \mathrm{M}$.

The ternary filter requires operation at an oversampled rate (OSR), and this will be met as the input signal is assumed to be a $\Sigma \Delta$ modulated bit-stream. The number of taps, $N$, is usually the same as the upsampling ratio, OSR. However, a higher value of $N$ could also be selected at the expense of increasing the delay of the filter, which is inversely related to the bandwidth. If $N$ is too large, the high frequency contents of the signal will be attenuated.

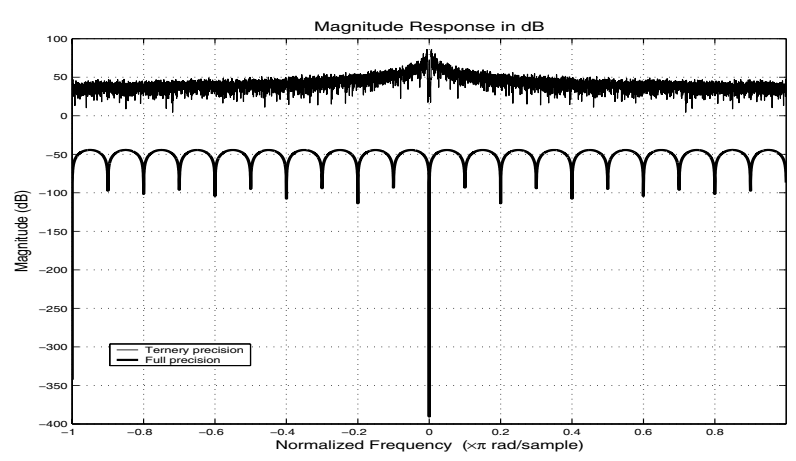

Fig. 4. Frequency response of the ternary filter in the proposed comb structure.

In our simulation, the proposed single-bit comb filter is designed, as an example, to attenuate the effect of the $10^{\text {th }}$-order harmonics in narrow-band signals transmitted over powerlines. A direct form FIR filter is designed for this purpose and used as a target impulse response.

Fig.(4) shows the frequency response of the FIR filter with full precision and ternary precision coefficients $(\mathrm{OSR}=128)$. The simulated frequency response of the single-bit comb filter is shown in Fig.(5). The gain factor $b$ is out of our interest here and is assumed to be constant equal to 0.001 throughout this work. The phase response of the filter system can be seen if 


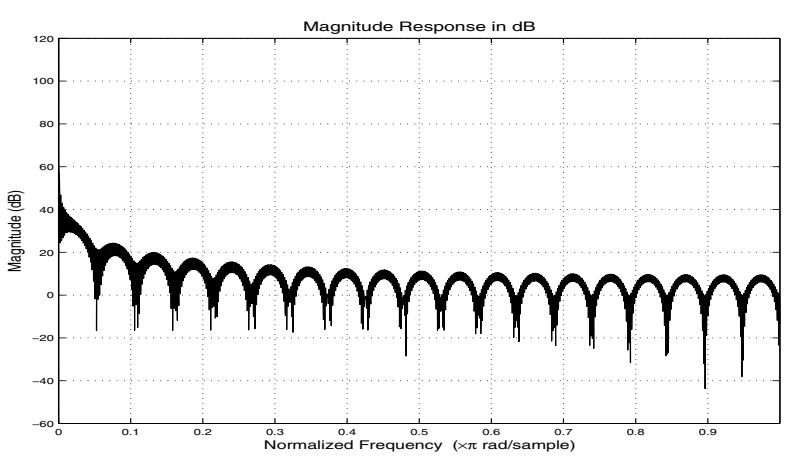

Fig. 5. Frequency Response of the proposed ternary- $\Sigma \Delta$ single-bit comb filter with OSR $=128$.

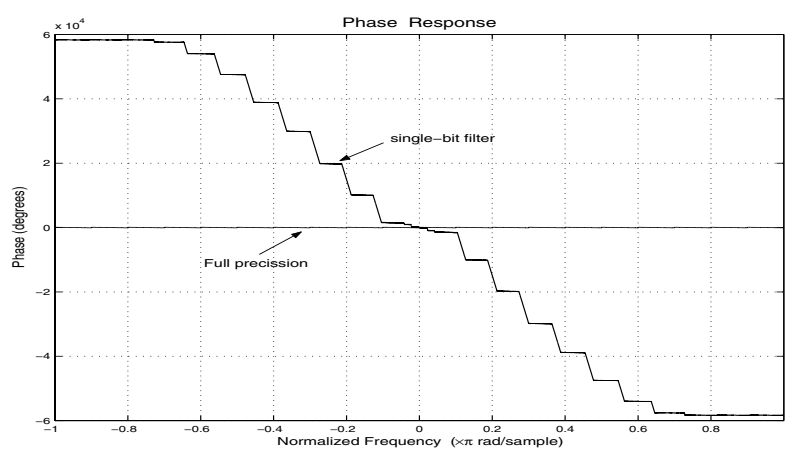

Fig. 6. Phase response of the proposed single-bit ternary- $\Sigma \Delta$ comb filter.

Fig.(6). The non-linear effect of the $\Sigma \Delta \mathrm{M}$ stage on the phase performance of the overall combination is apparent.

\section{A Sigma-Delta Comb Filter}

A comb filter can be created by taking an FIR filter with the following system function [7]

$$
H(z)=\sum_{k=0}^{M} h(k) z^{-k}
$$

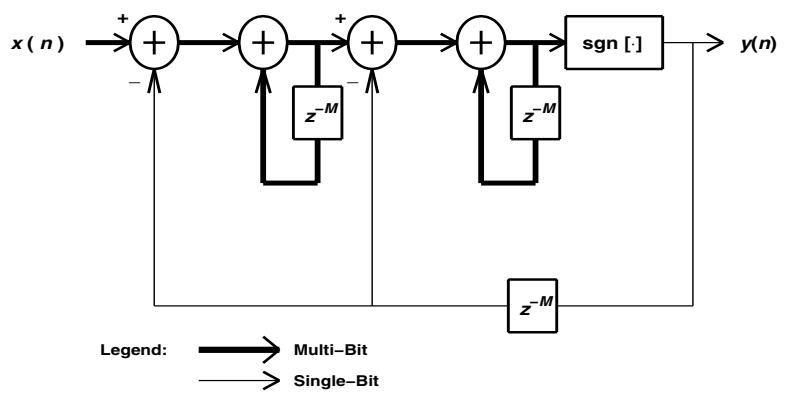

Fig. 7. Block diagram of the proposed $\Sigma \Delta \mathrm{M}$ structure.

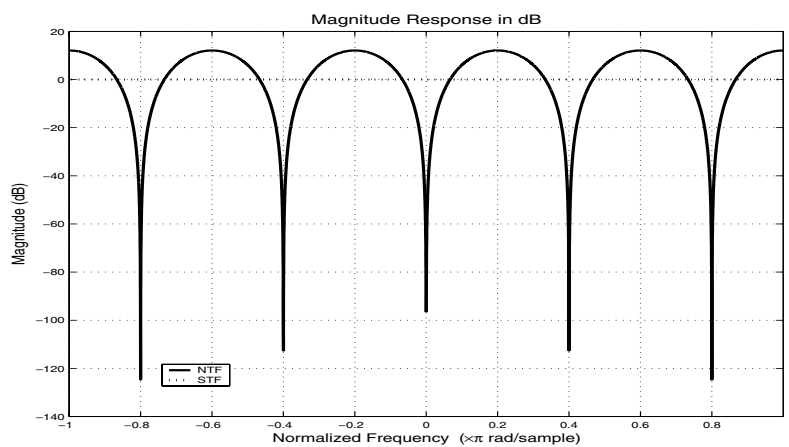

Fig. 8. Noise and signal transfer functions of the proposed $\Sigma \Delta$ single-bit comb filter.

then replacing $z$ by $z^{p}$, where $p$ is a positive integer. Thus, the new FIR filter has a system function as follows:

$$
H_{c}(z)=\sum_{k=0}^{M} h(k) z^{-k p} .
$$

If the frequency response of the original FIR filter is $H\left(e^{j \omega}\right)$, the frequency response of the new FIR in (11) is given by

$$
H_{c}\left(e^{j \omega}\right)=\sum_{k=0}^{M} h(k) z^{-j k p \omega}
$$

i.e.,

$$
H_{c}\left(e^{j \omega}\right)=H\left(e^{j p \omega}\right) .
$$

Consequently, the frequency response $H_{c}\left(e^{j \omega}\right)$ is simply $p$ order repetition of $H\left(e^{j \omega}\right)$ in the range $0 \leq \omega \leq 2 \pi$. Hence, if we replace $z^{-1}$ by $z^{-M}$ directly in the second-order $\Sigma \Delta \mathrm{M}$ noise transfer function as shown in the Fig. (7), the transfer function of the corresponding discrete-time linear model can be given as follows [8]

$$
H(z)=X(z)+E(z)\left(1-z^{-M}\right)^{2} .
$$

The noise-shaping filter, $\left(1-z^{-M}\right)^{2}$, is a comb filter with notches at frequencies $2 \pi k / M$, where $k=0,1,2, \cdots, M-1$. This filter can be used for any signal that has narrow-band frequency components in these locations. Fig. (8) shows the signal transfer function, $X(z)$, and the noise transfer function, $E(z)$, according to (14).

The simulated frequency response of this structure (using the same sampling rate and design requirements as in the ternary- $\Sigma \Delta$ structure) is shown in Fig. (8). Fig. (9) depicts the phase response of this structure.

From a hardware viewpoint, both of the proposed structures for digital comb filtering have the advantage of simple implementation, as there are no multi-bit addition or multiplication operations in their structure, however, the second structure is even simpler to construct and proved to have better response in high-frequency applications.

The proposed structures lend themselves well in broadband PLC applications, as they are efficient in hardware implementation with high performance. 


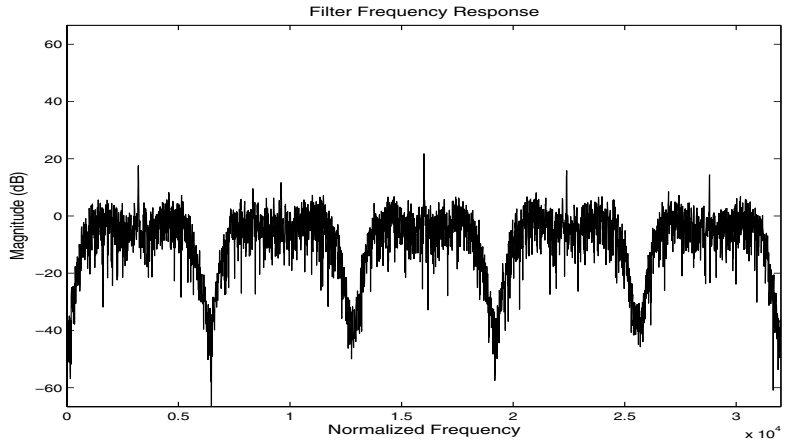

Fig. 9. Frequency Response of the proposed $\Sigma \Delta$ single-bit comb filter.

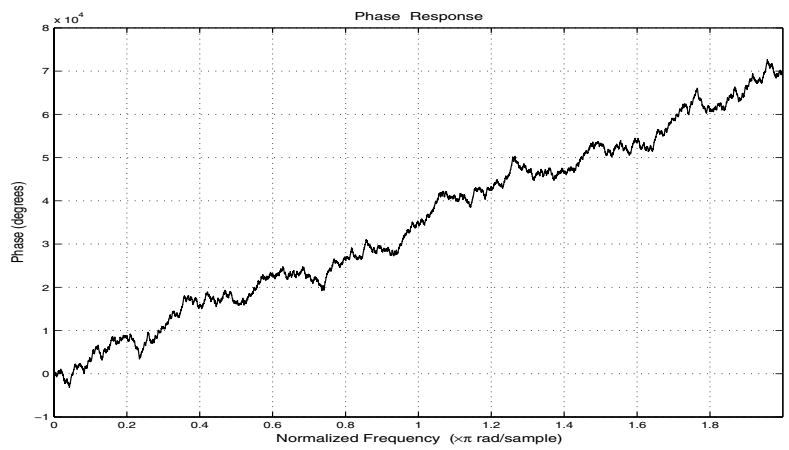

Fig. 10. Phase Response of the proposed $\Sigma \Delta$ single-bit comb filter.

\section{Stability of the Proposed Structures}

Linear analysis has been used to model the quantization noise in $\Sigma \Delta$ systems [9]. Though useful, the linear model is unable to model the system well enough to predict the stability and performance for a given design. This is due to the nonlinear behavior of the $\Sigma \Delta$ systems. However, attempts to better predict the behavior of $\Sigma \Delta \mathrm{M}$ using non-linear analysis techniques have produced promising results [10]. Initial simulation results showed that the proposed structures are stable, however, full analysis based on non-linear analysis will be handled in future works.

\section{CONCLUSION}

Two structures for single-bit digital comb filtering are proposed and simulated. In the first structure, a comb filter is designed based on ternary filtering such that both the input signal and the target impulse response are encoded using a $\Sigma \Delta$ modulator. The second structure is based on a secondorder $\Sigma \Delta$ modulator. The frequency response obtained in both cases is very near to the required response of a comb filter. The proposed filters can be built using simple hardware, and hence they are potentially suitable for VLSI implementation. They are also suitable for broadband applications such as power-line communications.

\section{ACKNOWLEDGEMENT}

This work is supported by the Australian Research Council under the ARC Discovery Grant DP0557429.

\section{REFERENCES}

[1] N. Benvenuto, L. E. Franks, and F. S. Hill, JR., "Realization of finite impulse response filters using coefficients $+1,0,-1$, IEEE Transactions on Communications, vol. COM-33, no. 10, pp. 1117-1125, Oct. 1985.

[2] A. C. Thompson, P. O'Shea, Z. M. Hussain, and B. R. steele, "Efficient single-bit ternary digital filtering using sigma-delta modulator," IEEE Signal Processing Letters, vol. 11, no. 2, pp. 162-166, Feb. 2004.

[3] B. R. Steele, Efficient Signal Processing Through the Use of Sigma-Delta Modulation and Ternary Filters, $\mathrm{PhD}$ dissertation, RMIT University, Melbourne, 2003.

[4] A. C. Thompson, Techniques in Single-Bit Digital Filtering, $\mathrm{PhD}$ dissertation, RMIT University, Melbourne, 2004.

[5] J. J. Paulos, G. T. Brauns, M. B. Steer, and S. H. Ardalan, "Improved SRN using trilevel delta-sigma modulator," IEEE International Symposium on Circuits and Systems, Philadliphia, PA, May 1987.

[6] S. S. Abeysekera, and K. P. Padhi, "Design of multiplier free FIR filters using a LADF sigma-delta modulator," IEEE International Symposium on Circuits and Systems, Geneva, Switzerland, May 2000.

[7] J. G. Proakis and D. G. Manolakis, Digital Signal Processing: Principles, Algorithms, and Applications, Third Edition, Printice-Hall, 1996.

[8] K. Chuan, G. E. Sobelman, E. Saberinia, and A. H. Tewfik, "Performance of N-tone sigma-delta modulation for UWB-OFDM," IEEE Communications Society, pp. 1483-2486, 2004.

[9] P. W. Wong, "Fully sigma-delta modulation encoded FIR filters," IEEE Transactions on Signal Processing, vol. 40, no. 6, Jun. 1992.

[10] P. Steiner and W. Yang, "Stability analysis of the second order $\Sigma \Delta$ modulator," IEEE International Symposium on Circuits and Systems (ISCAS'94),vol. 5, pp. 365 - 368, 1994. 